MATHEMATICS OF COMPUTATION

Volume 66, Number 220, October 1997, Pages 1555-1560

S 0025-5718(97)00897-1

\title{
B-SPLINES AND OPTIMAL STABILITY
}

\author{
J.M. PEÑA
}

\begin{abstract}
It is proved that, among all nonnegative bases of its space, the B-spline basis is optimally stable for evaluating spline functions.
\end{abstract}

\section{INTRODUCTION}

In some recent papers [3]-[5], several optimal properties of the B-spline basis have been studied. Different viewpoints have been considered; for instance, the shape preserving properties in Computer Aided Geometric Design (see [4]) or the supports of the basis functions (see [3]). The B-spline basis $b=\left(b_{0}, \ldots, b_{n}\right)$ is a normalized nonnegative basis, that is $b_{i} \geq 0 \forall i=0, \ldots, n$, and $\sum_{i=0}^{n} b_{i}=1$. The interest of normalized nonnegative bases of a space comes from their convex hull property. In Computer Aided Geometric Design, this property implies that, for any control polygon, the corresponding curve always lies in the convex hull of the control polygon. In this paper, we shall prove a property of the optimal stability of the B-spline basis among all nonnegative bases of its space.

Given a basis $u=\left(u_{0}, \ldots, u_{n}\right)$ of a real vector space $U$ of functions defined on $\Omega$ and a function $f \in U$, there exists a unique sequence of real coefficients $\left(c_{0}, \ldots, c_{n}\right)$ such that

$$
f(t)=\sum_{i=0}^{n} c_{i} u_{i}(t)
$$

for all $t \in \Omega$. One practical aspect to consider in the evaluation of the function $f$ is the stability with respect to perturbations of the coefficients, which depends on the chosen basis of the space. We want to know how sensitive a value $f(t)$ is to random perturbations of a given maximum relative magnitude $\varepsilon$ in the coefficients $c_{0}, \ldots, c_{n}$ corresponding to the basis. Following [7] and [6], we can bound the corresponding perturbation $\delta f(t)$ of the change of $f(t)$ by means of a condition number

$$
C_{u}(f(t)):=\sum_{i=0}^{n}\left|c_{i} u_{i}(t)\right|,
$$

for the evaluation of $f(t)$ in the basis $u$ :

$$
|\delta f(t)| \leq C_{u}(f(t)) \varepsilon .
$$

Received by the editor May 10, 1995 and, in revised form, July 29, 1996.

1991 Mathematics Subject Classification. Primary 65D07, 41A15.

Key words and phrases. B-splines; optimal stability, condition number, nonnegative matrices, partial ordering.

This work was partially supported by the Spanish Research Grant DGICYT PB93-0310 and by the EU project CHRX-CT94-0522.

(C) 1997 American Mathematical Society 
Let us observe that $C_{u}(f(t))$ depends on the basis $u$, on the function $f$ and on the point $t$. We shall consider that a basis $b$ is optimally stable among the nonnegative bases of its space $U$ if there does not exist any other basis $u$ of nonnegative functions such that $C_{u}(f(t)) \leq C_{b}(f(t))$ for each function $f \in U$ evaluated at every $t$. In general, existence or uniqueness of optimally stable bases cannot be guaranteed. In [6] it was proved that the Bernstein basis is optimally stable among all nonnegative bases of the space of polynomials of degree less than or equal to $n$. In Section 2 (in Theorem 2.3) we prove a similar result (with respect to the corresponding space) for the B-spline basis. The customary condition number for the B-spline basis has been extensively considered in the literature (see, for instance, [1] or [8]).

Section 3 includes some remarks relating optimal stability of bases in general spaces with the minimal elements of a partial ordering among nonnegative bases of the space. This partial ordering was considered in [5] and [6]. In fact, in [5] it was related to the customary condition number of a basis. However, minimal elements for this partial order have not, in general, a minimal condition number among nonnegative bases, as we shall recall in Section 3.

\section{Optimal stability of the B-Spline Basis}

Let us introduce first the B-spline basis. Let $\Pi_{k-1}$ be the space of polynomials of degree less than or equal to $k-1$. Let $\Delta=\left\{\theta_{1}, \ldots, \theta_{l}\right\}$ be a partition of the interval $I=[\alpha, \beta]$, where

$$
\alpha=\theta_{0}<\theta_{1}<\cdots<\theta_{l}<\theta_{l+1}=\beta,
$$

and let $I_{i}=\left[\theta_{i}, \theta_{i+1}\right], i=0, \ldots, l$. For any multiplicity vector $m=\left(m_{1}, \ldots, m_{l}\right)$ of positive integers such that $m_{i} \leq k-1, i=1, \ldots, l$, let us define

$$
\begin{aligned}
S_{k, \Delta, m} & =\left\{s: I \rightarrow \mathbb{R}|s|_{I_{i}} \in \Pi_{k-1}, i=0, \ldots, l ;\right. \\
& \left.s^{(j)}\left(\theta_{i}^{-}\right)=s^{(j)}\left(\theta_{i}^{+}\right), i=1, \ldots, l, j=0,1, \ldots, k-m_{i}-1\right\},
\end{aligned}
$$

the space of polynomial splines of order $k$ with knots $\theta_{1}, \ldots, \theta_{l}$ of multiplicities $m_{1}, \ldots, m_{l}$.

Now let us define the extended sequence of knots, that is, each knot $\theta_{i}$ is repeated $m_{i}$ times,

$$
\alpha=\tau_{0}=\cdots=\tau_{k-1}<\tau_{k} \leq \cdots \leq \tau_{n}<\tau_{n+1}=\cdots=\tau_{n+k}=\beta,
$$

where $n=k+m_{1}+\cdots+m_{l}-1$. It is well-known that the functions

$$
N_{i, k}(t):=\left(\tau_{i+k}-\tau_{i}\right)\left[\tau_{i}, \ldots, \tau_{i+k}\right](\cdot-t)_{+}^{k-1}, \quad i=0, \ldots, n,
$$

form a basis of $S_{k, \Delta, m}$ called the B-spline basis. Besides, the supports of the Bsplines are $\operatorname{supp} N_{i, k}=\left[\tau_{i}, \tau_{i+k}\right]$, and we also have that $N_{i, k}(t)>0$ for all $t \in$ $\left(\tau_{i}, \tau_{i+k}\right)$ and $\sum_{i=0}^{n} N_{i, k}=1$.

We shall assume that we have a given space $S_{k, \Delta, m}$ and we shall simplify our notation by denoting with $b=\left(b_{0}, \ldots, b_{n}\right)$ the corresponding B-spline basis

$$
b_{i}:=N_{i, k} .
$$

In the next auxiliary result we shall use the previous notations. 
Lemma 2.1. The B-spline basis $b=\left(b_{0}, \ldots, b_{n}\right)$ satisfies

$$
\lim _{t \rightarrow \tau_{j}^{+}} \frac{b_{i}(t)}{b_{j}(t)}=0, \quad \text { if } i>j,
$$

and

$$
\lim _{t \rightarrow \tau_{j+k}^{-}} \frac{b_{i}(t)}{b_{j}(t)}=0, \quad \text { if } i<j .
$$

Proof. Let us recall (see for instance Theorem 4.17 of [9]) that the B-spline function $b_{i}$ satisfies

$$
b_{i}^{(j)}\left(\tau_{i}^{+}\right)=0, \quad j=0,1, \ldots, k-\nu_{i}-2 \text { and } b_{i}^{\left(k-\nu_{i}-1\right)}\left(\tau_{i}^{+}\right) \neq 0,
$$

and

$$
b_{i}^{(j)}\left(\tau_{i+k}^{-}\right)=0, \quad j=0,1, \ldots, k-\mu_{i}-2 \text { and } b_{i}^{\left(k-\mu_{i}-1\right)}\left(\tau_{i+k}^{-}\right) \neq 0,
$$

where $\nu_{i}$ and $\mu_{i}$ are given by

$$
\begin{gathered}
\nu_{i}=\max \left\{j \mid \tau_{i}=\tau_{i+1}=\cdots=\tau_{i+j-1}\right\}, \\
\mu_{i}=\max \left\{j \mid \tau_{i+k}=\tau_{i+k-1}=\cdots=\tau_{i+k-j+1}\right\} .
\end{gathered}
$$

If $i \geq j+\nu_{j}$, then $\tau_{i}>\tau_{j}$ and so $b_{i}(t)=0, b_{j}(t)>0$ for all $t \in\left(\tau_{j}, \tau_{j+\nu_{j}}\right)$, which implies (2.1). On the other hand, if $i<j+\nu_{j}$, we know from (2.3) that the restriction of $b_{i}$ to the interval $\left(\tau_{j}, \tau_{j+\nu_{j}}\right)$ is a polynomial which has a zero of higher order at $\tau_{j}$ than the restriction of $b_{j}$ to the same interval, which implies that (2.1) holds. Analogously we may show (2.2) using (2.4) instead of (2.3).

In order to prove the main theorem we also need the following matrix result.

Lemma 2.2. Let $M$ be an $n \times n$ nonsingular and nonnegative matrix such that the first nonzero entry of each column of $M^{-1}$ is positive. Then $M^{-1}$ is, up to permutation of columns, a lower triangular matrix.

Proof. We shall prove, by induction on $n$, that $M^{-1}=L P$, where $L$ is a nonsingular lower triangular matrix and $P$ is a permutation matrix. The result is trivial for $n=1$. Let us assume that the result holds for $n-1$ and we shall prove it for $n$. By hypothesis, $M^{-1}$ has a nonnegative first row. Let us assume that this row has at least two nonzero entries: the $(1, i)$ and $(1, j)$ entries, with $i \neq j$. Then, from $M^{-1} M=I$ and the nonnegativity of $M$, we find that the $(i, k)$ and $(j, k)$ entries of $M$ are zero for all $k>1$, in contradiction with the nonsingularity of $M$. Thus $M^{-1}$ has a unique nonzero element in the first row and so there exists a permutation matrix $Q$ such that the first row of $M^{-1} Q$ has its nonzero element in the first place. Clearly, the submatrix formed by the last $n-1$ rows and columns of $M^{-1} Q$ is invertible, and let us denote it by $B^{-1}$. Since $B$ is an $(n-1) \times(n-1)$ nonnegative and nonsingular matrix such that the first nonzero entry of each column of $B^{-1}$ is positive, we deduce by the induction hypothesis that $B^{-1}=L_{1} P_{1}$, where $L_{1}$ is a nonsingular lower triangular matrix and $P_{1}$ is a permutation matrix. Thus $M^{-1}=L P$, with $L$ a nonsingular lower triangular matrix and $P$ a permutation matrix.

Now, using the previous lemmas, we can prove the main theorem of the paper. 
Theorem 2.3. The B-spline basis b is optimally stable with respect to the set of nonnegative bases of $S_{k, \Delta, m}$, that is, there does not exist (up to permutation or positive scaling) a basis of nonnegative functions $u=\left(u_{0}, \ldots, u_{n}\right)$ of $S_{k, \Delta, m}$ such that

$$
C_{u}(f(t)) \leq C_{b}(f(t))
$$

for each spline function $f \in S_{k, \Delta, m}$ evaluated at every value $t \in[\alpha, \beta]$.

Proof. Let us assume that there exists a nonnegative basis $u$ satisfying $(2.5)$ for each spline function $f \in S_{k, \Delta, m}$ evaluated at every value $t \in[\alpha, \beta]$. Let $A=\left(a_{i j}\right)_{0 \leq i, j \leq n}$ be the matrix of change of basis

$$
b=u A .
$$

It is sufficient to prove that $A$ is a generalized permutation matrix, i.e. a matrix with the same zero pattern as a permutation matrix.

Let us see first that $A$ is a nonnegative matrix. Let us assume that $a_{p q}<0$ for some $p, q$. Since $u_{p}$ is a nonnegative basis function, there exists $t \in[\alpha, \beta]$ such that $u_{p}(t)>0$. Then, since by $(2.6)$

$$
b_{q}=a_{0 q} u_{0}+\cdots+a_{p q} u_{p}+\cdots+a_{n q} u_{n}
$$

and $b_{q}(t) \geq 0$, we deduce that there exists $m$ such that $a_{m q}>0$ and $u_{m}(t)>0$. Thus, taking into account that $a_{p q}$ and $a_{m q}$ are of opposite sign and the functions in $u$ are nonnegative, we may deduce that

$$
C_{u}\left(b_{q}(t)\right)=\sum_{i=0}^{n}\left|a_{i q}\right| u_{q}(t)>\left|\sum_{i=0}^{n} a_{i q} u_{q}(t)\right|=\left|b_{q}(t)\right|=C_{b}\left(b_{q}(t)\right) .
$$

This contradiction proves that $A$ must be nonnegative.

Let us see now that the first and last nonzero entry of each column of $A^{-1}$ is positive. By $(2.6), u=b A^{-1}$. Given any $j \in\{0, \ldots, n\}$, we have to prove that, if $u_{j}=\sum_{i=0}^{n} \alpha_{i} b_{i}$, then the first and last nonzero coefficients in the sequence $\left(\alpha_{0}, \ldots, \alpha_{n}\right)$ must be positive. Let $\alpha_{k}$ be the first nonzero coefficient in the sequence $\left(\alpha_{0}, \ldots, \alpha_{n}\right)$. From $(2.1)$ we may deduce that

$$
\lim _{t \rightarrow \tau_{k}^{+}} \frac{u_{j}(t)}{b_{k}(t)}=\alpha_{k}
$$

Since $u_{j}$ and $b_{k}$ are nonnegative functions, we conclude that $\alpha_{k}$ must be nonnegative and therefore positive. Applying an analogous reasoning using (2.2) instead of (2.1), we may deduce that the last nonzero coefficient in the sequence $\left(\alpha_{0}, \ldots, \alpha_{n}\right)$ must also be positive.

We have already seen that $A$ is nonnegative and the first and last nonzero entry of each column of $A^{-1}$ are positive. By Lemma 2.2 there exist a permutation matrix $P$ and a lower triangular matrix $L$ such that $A^{-1}=L P$. Let $C$ be the nonsingular upper triangular matrix obtained by reversing the order of the rows and the columns of $L=A^{-1} P^{T}$. Since the last nonzero entry of each column of $A^{-1}$ is positive, the same property holds for $A^{-1} P^{T}$, and therefore the first nonzero entry of each column of $C$ is positive. It can be easily checked that $C^{-1}$ is nonnegative. Applying now Lemma 2.2 to $C^{-1}$, we deduce that there exists a permutation of the columns of the nonsingular upper triangular matrix $C$ such that the resulting matrix is also lower triangular (and nonsingular). This implies that $C$ is a diagonal matrix. In consequence, $L=A^{-1} P^{T}$ is also a diagonal matrix. Therefore $A$ can be written as 
the product of a permutation matrix $P^{T}$ and a diagonal matrix $L^{-1}$, and so $A$ is a generalized permutation matrix.

\section{SOME GENERAL REMARKS}

The problem of finding optimally stable nonnegative bases is closely related to finding minimal elements for a partial order $\preceq$ among nonnegative bases of the space. Let $u=\left(u_{0}, \ldots, u_{n}\right), v=\left(v_{0}, \ldots, v_{n}\right)$ be two bases of a vector space $U$ of functions defined on $\Omega \subseteq \mathbb{R}^{s}$. Then we say that $u \preceq v$ if there exists a nonnegative matrix $A$ such that $v=u A$. It is easy to see that the fact that $u \preceq v$ and $v \preceq u$ are satisfied simultaneously is equivalent to saying that $u$ and $v$ are identical up to permutation and scaling. Taking into account that the product of nonnegative matrices is a nonnegative matrix, it follows that $\preceq$ induces a partial ordering among the bases up to permutation and scaling. This partial ordering has been recently used in [5] and [6].

If $u, v$ are two nonnegative bases of a space $U$ such that $u \preceq v$, we deduce from the definition of $\preceq$ and the nonnegativity of $u, v$ and $A$ that $C_{u}(f(t)) \leq C_{v}(f(t))$ for each function $f \in U$ evaluated at every $t \in \Omega$. One can also observe, using the argument of the second paragraph of the proof of Theorem 2.3 (which is a slight refinement of the proof of the converse of Theorem 1 of [6]), that $u \preceq v$ if and only if $C_{u}(f(t)) \leq C_{v}(f(t))$ for each function $f \in U$ evaluated at every $t \in \Omega$. Thus, the problem of finding optimally stable nonnegative bases reduces to finding minimal elements with respect to $\preceq$ among the nonnegative bases of the space.

In the framework of spaces of functions with totally positive bases, several characterizations of minimal elements with respect to $\preceq$ were given in Theorem 3.8 of [5]. Let us recall that a matrix is totally positive if all its minors are nonnegative and that a system of functions $u=\left(u_{0}, \ldots, u_{n}\right)$ defined on $I \subseteq \mathbb{R}$ is called totally positive if all its collocation matrices

$$
M\left(\begin{array}{c}
u_{0}, \ldots, u_{n} \\
t_{0}, \ldots, t_{m}
\end{array}\right):=\left(u_{j}\left(t_{i}\right)\right)_{i=0, \ldots, m ; j=0, \ldots, n},
$$

where $t_{0}<\cdots<t_{m}$ in $I$, are totally positive. Totally positive bases are especially useful in Computer Aided Geometric Design due to their shape preserving properties (see, for instance, [2]).

For a normalized basis $u=\left(u_{0}, \ldots, u_{n}\right)$ (i.e., with $\sum_{i=0}^{n} u_{i}=1$ ) of a vector space $U$ of functions defined on $\Omega \subseteq \mathbb{R}^{s}$, we considered in [5] the number

$$
\kappa(u):=\left(\min _{\left\|\left(c_{0}, \ldots, c_{n}\right)\right\|_{\infty}=1} \sup _{x \in \Omega}\left|\sum_{i=0}^{n} c_{i} u_{i}(x)\right|\right)^{-1},
$$

as the condition number, as usual. The condition number $\kappa(u)$ depends only on the basis $u$, in contrast to $C_{u}(f(t))$, which depends on the basis $u$, on the function $f$ and on the point $t$. It is well-known (see for instance Section 4 of [5]) that $\kappa(u$ ) satisfies

$$
\kappa(u)^{-1} \max _{i=0, \ldots, n}\left|c_{i}\right| \leq \sup _{x \in \Omega}\left|\sum_{i=0}^{n} c_{i} u_{i}(x)\right| \leq \max _{i=0, \ldots, n}\left|c_{i}\right|
$$

for any function $\sum_{i=0}^{n} c_{i} u_{i}$ in $U$. Therefore, the condition number $\kappa$ can be considered as a measure of the efficiency of the basis for the process of controlling a function through its coefficients. Although for this condition number it is also true 
that if $u \preceq v$, then $\kappa(u) \leq \kappa(v)$ (see Proposition 4.2 of [5]), in this case minimal (nonnegative) bases with respect to $\preceq$ are not necessarily minimally conditioned among the nonnegative bases of $U$. For instance, we saw in Example 4.4 of [5] that, although the Bernstein basis $b=\left(b_{0}, b_{1}, b_{2}\right)$ is minimal for $\preceq$ (see also Corollary 1 of [6]), there exists a basis of the space of polynomials on $[0,1]$ of degree less than or equal to 2 formed by nonnegative functions $u=\left(u_{0}, u_{1}, u_{2}\right)$, with $u_{0}(t)=(3 t / 2-1)^{2}, u_{1}(t)=3 t(1-t)$ and $u_{2}(t)=3 t^{2} / 4$, whose condition number $\kappa(u)$ is strictly smaller than $\kappa(b)$.

\section{ACKNOWLEDGEMENT}

I wish to thank Dr. J.M. Carnicer for his helpful ideas, and Dr. M. Floater for improving the readability of the paper.

\section{REFERENCES}

[1] C. de Boor, The exact condition of the B-spline basis may be hard to determine, J. Approx. Theory 60 (1990), 334-359. MR 91h:65023

[2] J. M. Carnicer and J. M. Peña, Shape preserving representations and optimality of the Bernstein basis, Adv. Comput. Math. 1 (1993), 173-196. MR 94i:65138

[3] J. M. Carnicer and J. M. Peña, Least supported bases and local linear independence, Numer. Math. 67 (1994), 289-301. MR 95d:65012

[4] J. M. Carnicer and J. M. Peña, Totally positive bases for shape preserving curve design and optimality of B-splines, Comput. Aided Geom. Design 11 (1994), 633-654. MR 95i:65033

[5] J. M. Carnicer and J. M. Peña, Total positivity and optimal bases, Total positivity and its applications (M. Gasca, C.A. Micchelli, eds.), Kluwer Academic Press, Dordrecht, 1996, pp. 133-155. CMP 97:04

[6] R. T. Farouki and T. N. T. Goodman, On the optimal stability of the Bernstein basis, Math. Comp. 65 (1996), 1553-1566. MR 97a:65021

[7] R. T. Farouki and V. T. Rajan, On the numerical condition of polynomials in Bernstein form, Comput. Aided Geom. Design 4 (1987), 191-216. MR 89a:65028

[8] T. Lyche, Condition Numbers for B-splines, Numerical Analysis 1989 (D. F. Griffiths and G. A. Watson, eds.), Longman Scientific and Technical, Essex, 1990, pp. 182-192. CMP 91:17

[9] L. L. Schumaker, Spline Functions: Basic Theory, John Wiley and Sons, New York, 1981. MR 82j:41001

Departamento de Matemática Aplicada, Universidad de Zaragoza, 50009 Zaragoza, SPAIN

E-mail address: jmpena@posta.unizar.es 